\title{
Comprensión de las medidas de tendencia central: un estudio comparativo en estudiantes de pedagogía en matemática en dos instituciones formadoras chilenas ${ }^{1}$
}

\author{
Francisco Enrique Rodríguez Alveal \\ Ana Carolina Maldonado Fuentes \\ Pedro Rodrigo Sandoval Rubilar
}

Resumen: Producto de la abundancia de información del tipo estadística presente en la vida cotidiana se ha originado un interés por la formación estadística, particularmente a nivel de alumnos en formación inicial docente. En coherencia con lo anterior el objetivo del presente artículo es describir el dominio de las habilidades de descodificación en información presente en enunciados problemáticos como en representaciones gráficas mediante medidas de tendencia central. Para tal efecto se realizó una evaluación de tipo inferencial comparativa de corte transversal, mediante un muestreo estratificado a 73 estudiantes de Pedagogía en Matemática de dos universidades del Consejo de Rectores del centro sur del país, la primera de estatus jurídico público y la segunda privada. Se aplicó un instrumento debidamente validado por experto y de manera estadística arrojando un Alfa de Cronbach $\alpha=0,88$ y un índice de validez de contenido de 0,71 . En el análisis de la información se utilizaron métodos descriptivos numéricos (promedio aritmético, desviación estándar y frecuencia porcentual) y métodos inferenciales (prueba de comparaciones de proporciones). Los resultados evidencian que en general, los alumnos en Formación Inicial Docente (FID) presentan porcentajes de logro descendidos en relación al cálculo de medidas de resumen numéricas, tales como promedio aritmético o promedio ponderado, cuando los enunciados son de carácter implícito, como así también en lo referido a la simulación de distribuciones de datos bajo condiciones iniciales (promedio conocido). De lo anterior se puede inferir que los estudiantes en general no presentan conocimientos teóricos estadísticos en el uso de las medidas de tendencia central en relación con la naturaleza de las variables.

Palabras claves: Medidas de tendencia central. Formación de profesores. Evaluación de habilidades estadísticas.

\section{Understanding measures of central tendency: a comparative study on student teachers in mathematics in two Chilean educational institutions}

Abstract: Proceeds from the abundance of statistical information type present in everyday life has originated an interest in statistical training, particularly at the level of students in initial teacher training. Consistent with the above, the purpose of this article is to describe the domain of decoding skills in information in problematic statements and graphical representations using measures of central tendency. For this purpose, a comparative evaluation of inferential cross-section was performed, using a stratified sample of 73 students of Pedagogy in Mathematics from two universities of the Council of Rectors of the center-south, the first legal status of public and private second. An instrument duly validated by expert and statistically throwing a Cronbach's alpha $\alpha=0.88$ and

1 Investigación Financiada por Dirección de Investigación Universidad del Bio-Bío (DIUBB) código. 142623 4/R "Desarrollo de habilidades de análisis e interpretación de datos de tipo descriptivo en estudiantes de formación inicial docente: Un estudio comparativo entre dos instituciones formadoras" 
content validity index of 0.71 was applied. In the numerical analysis of information descriptive methods (arithmetic mean, standard deviation and frequency percentage) and inferential methods (comparison test of proportions) they were used. The results show that in general, students in Initial Teacher Training (FID) have descended achievement rates in relation to the calculation of numerical summary measures, such as average weighted arithmetic average or when the statements are implicit character, as well as with regard to the simulation data distributions under (known average) initial conditions. From the above it can be inferred that students usually do not provide statistical theoretical knowledge in using measures of central tendency in relation to the nature of the variables

Key words: Measures of central tendency.Teacher initial preparation. Statistical skills assessment.

Compreender as medidas de tendência central: um estudo comparativo sobre os professores dos alunos em matemática em duas instituições educacionais chilenas

Resumo: O produto da abundância do tipo de informação estatística presente na vida cotidiana levou a um interesse na formação estatística, em especial ao nível dos estudantes em formação inicial de professores. Consistente com o acima exposto, o objetivo deste artigo é descrever o domínio das competências na decodificação de informações em declarações problemáticas e representações gráficas usando medidas de tendência central. Para este efeito, uma avaliação da secção transversal comparativo inferencial foi realizada utilizando amostragem estratificada de 73 estudantes de Pedagogia em Matemática de duas universidades do Conselho de Reitores centro sul do país, o primeiro estatuto jurídico pública e segunda privado. um instrumento devidamente validado por peritos e estatisticamente rendendo $\alpha$ alfa de Cronbach $=0,88$ foi aplicado eo conteúdo do índice de 0,71 validade. $\mathrm{Na}$ análise da informação numérica métodos descritivos (média aritmética, desvio padrão e porcentagem de frequência) e os métodos de inferência (comparações de teste de proporções) foram usados. Os resultados mostram que, em geral, os alunos de Formação Inicial de Professores (FID) têm taxas de realização baixou em relação ao cálculo de medidas numéricas de resumo, como a média aritmética média ponderada ou em que as declarações são de caráter implícito, bem como em referência às distribuições de dados de simulação (média conhecida sob condições iniciais). Do exposto pode-se inferir que os estudantes geralmente não fornecem o conhecimento teórico estatística utilizando medidas de tendência central em relação à natureza das variáveis

Palavras-chave: Medidas de tendência central. Formação de professores. Habilidades de avaliação estatística.

\section{Antecedentes}

Según la UNESCO (1997, p. 123) la enseñanza de la Estadística es fundamental para el ciudadano al favorecer la adquisición de conocimientos y habilidades que le permiten acceder a la sociedad de la información. En este sentido la estadística juega un rol fundamental para el ejercicio de la ciudadanía en un mundo cada vez más globalizado, debido a que el sujeto moderno es demandado a poseer competencias para leer e interpretar tablas y gráficos que se presentan en los medios informativos para la toma de decisiones (HOLMES, 1980); como así también aquella información que se resume mediante indicadores numéricos o representaciones gráficas (BEHAR; OJEDA, 2000). Lo anterior cobra mayor relevancia, toda vez que se aspira al desarrollo de una comunicación compleja y pensamiento experto en la ciudadanía (REICH, 2011).

En este contexto, el Ministerio de Educación de Chile (MINEDUC), durante el año 2012, aprobó un nuevo marco curricular para el Sistema Escolar chileno, 
Comprensión de las medidas de tendencia central: un estudio comparativo en estudiantes de pedagogía en matemática en dos instituciones formadoras chilenas

siendo los contenidos de Modelamiento de Datos (estadística) incorporados desde el primer año básico en el sector de Matemática, con la finalidad de desarrollar el razonamiento probabilístico, modelamiento de datos e interpretación de ellos, habilidades que son propias de la Estadística (MINEDUC, 2012a).

Consecuentemente con ello, se espera que los currículos para la Formación Inicial Docente en Chile (FID) se encuentren alineados con los Estándares en Educación Básica (MINEDUC, 2011) y en Educación Media (MINEDUC, 2012 b), por lo cual se debería propender a un manejo de los contenidos disciplinarios en el área estadística y especialmente, en lograr una alfabetización estadística por parte de los futuros docentes, que según Gal (2002, p. 2), estaría relacionado con dos componentes interrelacionados:

a) Capacidad para interpretar y evaluar críticamente la información, los argumentos apoyados en datos o los fenómenos estocásticos que las personas pueden encontrar en diversos contextos, incluyendo los medios de comunicación, pero no limitándose a ellos.

b) Capacidad para discutir o comunicar sus opiniones respecto a tales informaciones estadísticas cuando sea relevante.

Por otro lado, en la investigación educativa en el campo de la estadística, las medidas de resumen numérico, en particular las de tendencia central, han suscitado gran interés como se puede constatar en los trabajos de autores a nivel internacional (GALICIA, 2009; COBO, 2003); debido a que la media aritmética o promedio es uno de los conceptos matemáticos que se introducen desde los primeros años del currículo escolar en diferentes países y, por otro lado, dicha medida es un índice que presenta una gran complejidad para su comprensión. Por tal motivo varios autores abordan su enseñanza a través de investigaciones con estudiantes de todos los niveles del sistema educacional (MOCHÓN; TLACHY, 2003, p. 5-28), (GARCÍA; GARCÍA CRUZ, 2004) y (GARCÍA CRUZ; GARRET, 2008, p. 49-66).

No obstante lo anterior, según Alveal y Rubilar (2012), las investigaciones sobre la enseñanza, conocimiento y comprensión de las medidas aritméticas o promedios son escasos a nivel nacional, y por otro lado, hay mayor dificultad para encontrar estudios sobre los conocimientos y habilidades de dichos conceptos estadísticos en estudiantes en la Formación Inicial Docente (FID) a nivel país.

Además, considerando que nadie puede enseñar lo que no conoce o domina (HERNÁNDEZ; SANCHO, 1996; RODRÍGUEZ, 1995), resulta de relevancia e importancia saber, si los futuros Docentes en formación Inicial Docente, 
conocen y poseen las habilidades para representar e interpretar las medidas de tendencia central, tales como la media aritmética (promedio aritmético). En especial dado que en su futuro desempeño laboral no sólo deberá enseñar a calcular de manera algorítmica los índices de resumen numérico de información, sino que enseñar las habilidades para codificar y descodificar los mensajes entregados en dichas medidas de resumen. En otras palabras, según lo señalado por (GAL, 2002), se trata de que dominen y enseñen a "hacer hablar los datos" y dar cuenta del mensaje que guardan ellos detrás de los resúmenes numéricos.

En síntesis, el presente estudio pretende indagar cuán alfabetizados están los estudiantes en Formación Inicial Docente de dos universidades de regiones del centro sur del país pertenecientes al Consejo de Rectores de Chile $\left(\mathrm{CRUCH}^{2}\right)$, una de estatus jurídico público (Universidad 1) y otra privada (Universidad 2). Una característica distintiva de las carreras de Pedagogía en Educación Matemática en dichas instituciones son sus cinco años de acreditación vigentes, lo que evidencia una valoración positiva de la trayectoria formativa de sus estudiantes y los resultados esperados en coherencia con las demandas de aseguramiento de la calidad en la educación superior. Específicamente en su malla curricular se incorporan dos actividades curriculares en el área estadística; el primero vinculado a contenidos de estadística descriptiva y probabilidad y el segundo estadística inferencial hasta modelos de regresión lineal múltiple. En el contexto de las medidas de tendencia central, toda vez que de ello dependerá el tipo de alfabetización que realizarán con sus futuros estudiantes del sistema escolar cuando ejerzan como docentes de aula. Específicamente, el presente estudio, indaga sobre las habilidades para la descodificación de información (lectura de datos) en diferentes contextos, para el cálculo y simulación de medidas de tendencia central bajo condiciones iniciales tales como conocido un promedio generar una distribución de datos crudos o agrupados.

\section{Objetivo general}

Describir el dominio de las habilidades de descodificación de información, presente en enunciados problemáticos como en representaciones gráficas mediante medidas de tendencia central, para su desarrollo algorítmico y simulación de distribuciones de datos, en estudiantes de Pedagogía en Educación Matemática pertenecientes a dos universidades del Consejo de Rectores de Chile.

2 Consejo de Rectores de las Universidades Chilenas es una persona jurídica de derecho público, de administración autónoma, creado por ley el 14 de agosto de 1954, como un organismo de coordinación de la labor universitaria de la nación. 
Comprensión de las medidas de tendencia central: un estudio comparativo en estudiantes de pedagogía en matemática en dos instituciones formadoras chilenas

\section{Objetivos específicos}

a) Comparar porcentajes de logro en habilidades de cálculo de medidas de tendencia central, a partir de la descodificación de información explícita e implícita, en estudiantes de Formación Inicial Docente de Pedagogía en Matemática.

b) Caracterizar las habilidades de descodificación de información numérica presente en representaciones gráficas y utilización de las medidas de tendencia central en estudiantes de Pedagogía en Matemática

c) Comparar porcentajes de logro en habilidades de simulación de datos a partir de un promedio conocido en un conjunto de datos crudos y agrupados, en estudiantes de Formación Inicial Docente de Pedagogía en Matemática.

\section{Marco teórico}

Uno de los principales objetivos de la estadística descriptiva es sintetizar e interpretar información mediante métodos gráficos y/o numéricos de naturaleza cualitativa y/o cuantitativa. En particular las representaciones gráficas destinadas a resumir información cuantitativa, como histogramas, polígonos de frecuencia simple, gráficos de cajas y tallo-hoja, los cuales solamente permiten visualizar de una manera relativamente subjetiva las características de la distribución de datos tales como forma, tendencia central y variabilidad, no siendo posible "cuantificarlas". En razón de lo anterior, se hace necesario contar con índices numéricos ya sean de tendencia central, forma y variabilidad que permitan sintetizar información las cuales nos permiten realizar comparaciones numéricas objetivas entre distribuciones de datos cuantitativos.

En este sentido cabe señalar que Moore (1991) definió la estadística como la ciencia de los datos y señaló que el objeto de la estadística es el razonamiento a partir de datos empíricos, subrayando la importancia del contexto, siendo la distribución de ellos una de las características esenciales en el análisis estadístico que trata de predecir propiedades de un conjunto de datos y no de cada dato aislado (BAKKER; GRAVEMEIJER, 2004). Por tal motivo la enseñanza de la estadística pretende que los estudiantes desarrollen las habilidades de leer, analizar, criticar y hacer inferencias a partir de distribuciones de datos (SHAUGHNESSY, 2007).

En tal sentido las medidas numéricas permiten sintetizar información cuantitativa y/o cualitativa proporcionando un valor representativo (también llamado valor típico) del conjunto de datos. Entre las medidas de resumen 
más importantes y ampliamente utilizadas se encuentra la media aritmética (promedio aritmético) que se obtiene de sumar todas las observaciones y dividiendo esta suma por el número de observaciones (datos) que hay en un grupo. Dicho algoritmo fue introducido primeramente por astrónomos de Babilonia y extendido en la Edad Media por el astrónomo danés Tycho Brahe (PLACKETT, 1970), siendo conservado hasta nuestros días. Otras medidas numéricas de interés son la mediana y la moda las cuales son también aplicables a datos cuantitativos o información de naturaleza ordinal e información cualitativa respectivamente. La comprensión y aplicación de estas medidas de resumen constituyen un contenido de alta complejidad (COBO; BATANERO, 2004), toda vez que no siempre son comprendidas por los estudiantes de educación secundaria y educación terciaria, inclusive.

Por otro lado la importancia de estudiar estas medidas de resumen radica en el hecho que ellas son la base para la comprensión de muchos otros conceptos estadísticos de interés, como por ejemplo variables aleatorias y distribuciones estadísticas de probabilidad, dado que se caracterizan por las medidas de posición central y dispersión (BATANERO, 2000).

Estudios realizados por Batanero (2000) entregan evidencias de que los estudiantes en general no muestran una adecuada comprensión de las medidas de tendencia central; en particular, en la claridad de los algoritmos de cálculo que se deben utilizar dependiendo la estructura de los datos (agrupados y no agrupados), como así también en las propiedades que hay detrás de ellas. Strauss y Bichler (1988), investigaron el desarrollo evolutivo de la comprensión del promedio aritmético en alumnos de 8 a 12 años, encontrando que a un porcentaje importante de ellos les resultaban demasiado abstractas las siguientes propiedades, las cuales Batanero (2000) las denomina elementos intensivos:

a) La suma de las desviaciones de las observaciones respecto al promedio es cero.

b) El valor de la media puede ser un valor decimal, lo cual puede tener o no sentido en la variable considerada, es decir, no es una operación interna.

c) El promedio en física juega el rol de centro de masa.

d) Se debe tener en cuenta los valores nulos en el cálculo de la media aritmética.

e) La media aritmética es un representante de los datos a partir de los cuales fue calculada ello debido a que considera a todas las observaciones en su computo. 
Comprensión de las medidas de tendencia central: un estudio comparativo en estudiantes de pedagogía en matemática en dos instituciones formadoras chilenas

Por otro lado, Mevarech (1983) plantea una explicación a los errores de los alumnos en relación al cálculo del promedio, postulando que los estudiantes creen que el conjunto de datos con la operación promedio aritmético satisface los axiomas de clausura, asociatividad, elemento neutro y elemento inverso, es decir, constituye un grupo aditivo, situación que no es verdadera.

Como se mencionó anteriormente el promedio aritmético es un valor "típico" o "representativo" de los datos. Campbell (1974) observa que, debido a ello, se tiende a situar al promedio en el centro del recorrido de la distribución de los datos, propiedad que solamente es válida para distribuciones simétricas; en cambio cuando la distribución de los datos es muy asimétrica (asimetría positiva o negativa) el promedio aritmético se desplaza hacia uno de los extremos o colas de la distribución, es decir, es afectada por observaciones extremas y en este caso la mediana o la moda serían un valor más representativo del conjunto de datos, siempre que la distribución sea unimodal. En este mismo sentido Russell y Mokros (1991) enuncian las siguientes capacidades que deberían adquirir los alumnos:

a) Dado un conjunto de datos, comprender la necesidad de emplear un valor central y elegir el más adecuado.

b) Construir un conjunto de datos que tenga un promedio dado.

c) Comprender el efecto que, sobre los promedios (media, mediana o moda), tiene un cambio en todos los datos o parte de ellos.

Otro medida de tendencia central ampliamente utilizada es el promedio aritmético ponderado. Gattuso (1996) encontró que una de las dificultades en la resolución de problemas donde interviene este índice es el algoritmo de cálculo; dado que, los estudiantes no ponderan adecuadamente los valores al resolver problemas donde interviene el promedio ponderado y además cometen el error de suprimir los valores nulos, algo que continúa observándose hasta en la universidad. Al mismo tiempo, el autor advierte el uso de estrategias diferenciadas según nivel de educación, con impacto en los resultados. A medida que aumenta la edad de los alumnos y con una instrucción adecuada mejora el empleo del algoritmo de cálculo y los alumnos usan con mayor frecuencia notación y métodos algebraicos. Sin embargo, los problemas que requirieren un conocimiento conceptual más profundo son resueltos por un porcentaje mayor de alumnos de secundaria que por universitarios, debido a que los primeros emplearon estrategias intuitivas y los segundos se concentraron en fórmulas algebraicas o definiciones formales. 
Dentro de este conjunto de indicadores, otro operador que asigna un valor numérico a un conjunto de datos es la mediana, de la cual existen diferentes definiciones equivalentes en los textos escolares, siendo una de ellas "el promedio central de una serie de datos ordenados según magnitud" (BATANERO, 2000), lo cual hace pensar en los alumnos la idea que la mediana es equivalente al promedio aritmético generando así dificultades conceptuales referentes a este estadígrafo de resumen. Otra dificultad radica procedimentalmente en su cálculo, dado que el algoritmo no es único y depende del tipo de información, de la forma de presentación de los datos e incluso del número de observaciones. No obstante la mediana siempre puede obtenerse para datos cuantitativos como así también en presencia de datos cualitativos ordinales, situación que no ocurre con el promedio aritmético. Entre las propiedades algebraicas podemos mencionar que la mediana no satisface la condición de clausura, asociatividad, existencia de elemento neutro y elemento inverso, pero es conmutativa. En resumen un conjunto de datos junto con la operación la mediana no satisface los axiomas de un grupo algebraico.

Finalmente la moda, que está asociada al valor(es) más frecuente de un conjunto de datos, presenta el inconveniente de no ser única y en alguno casos no existe lo cual ha hecho no ser considerada frecuente como un índice de posición central en la síntesis de información. No obstante presenta la característica de poder ser utilizada en presencia de datos cuantitativos y/o cualitativos.

Cabe mencionar que estas medidas de tendencia central se encuentran relacionadas con la forma de la distribución de un conjunto de datos, es decir, si los datos presentan una distribución simétrica el promedio aritmético, la mediana y la moda coinciden en distribuciones unimodales. En cambio si los datos presentan una distribución asimétrica a la derecha estas medidas de tendencia central aparecen en el siguiente orden moda-mediana y promedio. A su vez cuando los datos presentan una asimetría a la izquierda el orden es promedio-mediana y moda (para distribuciones unimodales).

\section{Diseño metodológico}

\section{Diseño del estudio}

Estudio cuantitativo inferencial comparativo de corte transversal (MCMILLAN; SCHUMACHER, 2007) debido a que se comparan las habilidades presentes en alumnos de Formación Inicial Docente de la carrera de Pedagogía en Matemática de dos universidades del centro sur del País. 


\section{Población}

Estudiantes de Pedagogía en Matemática de dos universidades del centro sur del país pertenecientes al Consejo de Rectores de Universidades Chilenas (CRUCH). La población estaba compuesta por 142 estudiantes, de los cuales 82 corresponden a la Universidad 1 y 60 a la Universidad 2.

\section{Muestreo y tamaño muestral}

El estudio se realizó sobre la base de un muestreo estratificado con una afijación proporcional al tamaño de los estratos (Universidad 1 y Universidad 2). En el cálculo de la muestra se consideró un error de aproximadamente un $8 \%$, un nivel de confianza del 95,0\% y una varianza máxima lo cual arrojó un tamaño muestral de 73 alumnos en Formación Inicial Docente de las carreras de Pedagogía en Matemática, de los cuales de los cuales 42 eran alumnos de la Universidad 1 y 31 de la Universidad 2, pertenecientes al Consejo de Rectores de las Universidades Chilenas ubicadas en el centro sur del país (MCMILLAN; SCHUMACHER, 2007).

Un factor de control fue que el 100,0\% de los encuestados ingresaron a la educación superior vía Prueba de Selección Universitaria mecanismo de carácter nacional y obligatorio. En la siguiente tabla se muestra la distribución de los encuestados según género, edad y dependencia del colegio de procedencia. Se observa que mayoritariamente los estudiantes son de género masculino $80,5 \%$ (Universidad 1) y $67,7 \%$ (Universidad 2) respectivamente no existiendo diferencias significativas ( $\mathrm{p}$-valor $=0,20)$ entre los estudiantes de Pedagogía en Matemática de ambas casas de estudio. Similarmente en el caso de la procedencia de los estudiantes no se presentan diferencias significativas considerando las modalidades municipal y particular subvencionado ( $\mathrm{p}$-valor $=0,78$ )

\section{Tabla 1- Estadísticas básicas de los estudiantes encuestados según Universidad.}

\begin{tabular}{lcccc}
\hline & & Universidad 1 & Universidad 2 & p-Valor \\
\hline Genero & & & & \\
& Femenino & 19,5 & 32,3 & \\
Edad (años) & Masculino & 80,5 & 67,7 & 0,20 \\
Dependencia & & $21,0 \pm 4,5$ & $22,9 \pm 5,2$ & 0,04 \\
& & & & \\
\multicolumn{2}{c}{ P. Subvencionado } & 29,3 & 32,3 & 0,78 \\
& Municipal & 70,7 & 67,7 & \\
\hline
\end{tabular}


Solamente se presentan diferencias significativas entre las edades promedios de los estudiantes de la Universidad 1 y Universidad 2 ( $\mathrm{p}$-valor=0,04).

\section{Instrumento}

En la estructura del instrumento se tuvieron en cuenta la revisión los contenidos del eje de Datos y Azar presentes en los textos escolares del sector Matemática de Educación Básica y Enseñanza Media distribuidos de manera gratuita por el Ministerio de Educación de Chile (MINEDUC, 2009, 2012a, 2012b), como así también las habilidades explicitadas por Russell y Mokros (1991). En la siguiente tabla se muestra las especificaciones del instrumento:

Tabla 2 - Tabla de especificaciones del instrumento.

\begin{tabular}{lc}
\hline Habilidad & Número de Reactivos \\
\hline $\begin{array}{l}\text { Cálculo algorítmico del promedio (aritmético simple y pondera- } \\
\text { do) y mediana a partir de la descodificación de enunciados con } \\
\text { información explicita. }\end{array}$ & $\mathbf{6}$ \\
$\begin{array}{l}\text { Descodificación de información en representaciones gráficas para } \\
\text { calcular o estimar el promedio aritmético o mediana. }\end{array}$ & $\mathbf{3}$ \\
$\begin{array}{l}\text { Simulación de datos a partir de un promedio conocido y compara- } \\
\text { ción datos desde las medidas de tendencia central y variabili- } \\
\text { dad. }\end{array}$ & $\mathbf{5}$ \\
Total de reactivos & $\mathbf{1 4}$ \\
\hline
\end{tabular}

El instrumento, fue sometido a valoración de expertos cuyas aportes permitieron obtener un índice de validez de contenido de 0,71 y en el análisis de fiabilidad arrojo un coeficiente Alfa de Cronbach de $\alpha=0,88$. En consecuencia, el instrumento posee una buena consistencia interna, es decir, la información es considerada fiable para objetivos del estudio.

\section{Análisis de la información}

En el análisis de la información se consideraron estadística descriptiva univariada numérica (porcentajes, promedio aritmético y desviación típica) y estadística inferencial. Para el estudio de diferencias significativas en los porcentajes de logro se utilizó la prueba de comparación de proporciones y la prueba de Pearson, considerando un nivel de significación del $\alpha=0,05$. El procesamiento de la información fue realizado mediante el uso del software estadísticos de fuente libre PSPP y R. 
Comprensión de las medidas de tendencia central: un estudio comparativo en estudiantes de pedagogía en matemática en dos instituciones formadoras chilenas

\section{Resultados}

Los resultados se presentan en tres secciones, en coherencia con los objetivos del estudio. En primer lugar, se entrega información sobre los porcentajes de logro asociadas a las habilidades cálculo de medidas de tendencia central a partir de la descodificación de información explicita e implícita presente en enunciados; en segundo lugar, los porcentajes de logro asociados a las habilidades de descodificación de información numérica presentada en representaciones gráficas (gráficos de barras e histogramas) y utilización de medidas de tendencia central y finalmente los porcentajes de logro en habilidades de simulación de distribuciones de datos a partir de un promedio conocido en conjuntos de datos crudos y agrupados.

Habilidades de cálculo de medidas de tendencia central de información en enunciados del tipo explicito e implícito

Además de la lectura e interpretación de resúmenes gráficos otra de las habilidades esperadas en los alumnos de enseñanza básica, media y superior, es la referida a obtener resúmenes numéricos de información cuantitativa y/o cualitativa como por ejemplo el cálculo e interpretación de las llamadas medidas de tendencia central, en particular el promedio aritmético, la mediana y la moda ya sea en datos sin agrupar o agrupados (tablas de distribución de frecuencia).

Uno de los problemas usuales en estadística es la presencia de datos considerablemente diferentes a los otros datos, técnicamente llamados datos atípicos los cuales pueden ser producto de errores en la medición, transcripción errónea de información, o un dato no esperado. Por ejemplo la presencia de un dato más grande o pequeño que la mayoría de las observaciones, los cuales usualmente se conocen en la literatura estadística como outlier.

En la siguiente tabla se presentan los porcentajes de logro alcanzados por los estudiantes en relación a la habilidad del cálculo de las medidas de tendencia central según institución. Se observa que solamente un 14,3\% de los alumnos de la Universidad 1 calculan correctamente el promedio aritmético en presencia de un dato atípico, aumentando este porcentaje a un 20,0\% en la Universidad 2, sin observarse diferencias estadísticamente significativas. Lo anterior puede tener diversas explicaciones, siendo una de ellas el escaso hincapié del tratamiento de resumen de información en presencia de datos atípicos. 
Tabla 3 - Distribución de los porcentajes de logro en estudiantes de FID de Pedagogía en Matemática, en relación a las habilidades de cálculo de promedios y mediana con enunciados explícitos e implícitos en datos crudos y agrupados

\begin{tabular}{|c|c|c|c|}
\hline \multirow[b]{2}{*}{ Habilidades de } & & \multirow{2}{*}{$\begin{array}{c}\text { Universidad } 1 \\
\text { Porcentaje } \\
\end{array}$} & \multirow{2}{*}{$\begin{array}{c}\text { Universidad } 2 \\
\text { Porcentaje } \\
\end{array}$} \\
\hline & & & \\
\hline \multirow{2}{*}{$\begin{array}{l}\text { Cálculo de promedio aritmético con informa- } \\
\text { ción explícita con de datos atípicos }\end{array}$} & Logrado & $14,3^{\mathrm{a}}$ & $20,0^{a}$ \\
\hline & No Logrado & 85,7 & 80,0 \\
\hline \multirow{2}{*}{$\begin{array}{l}\text { Cálculo de promedio con información explí- } \\
\text { cita de datos agrupados. }\end{array}$} & Logrado & $45,2^{\mathrm{a}}$ & $73,3^{b}$ \\
\hline & No Logrado & 54,8 & 26,7 \\
\hline \multirow{2}{*}{$\begin{array}{l}\text { Cálculo de la mediana cuando el número } \\
\text { de observaciones es par, con enunciado } \\
\text { explícito. }\end{array}$} & Logrado & $76,2^{\mathrm{a}}$ & $73,3^{a}$ \\
\hline & No Logrado & 23,8 & 26,7 \\
\hline \multirow{2}{*}{$\begin{array}{l}\text { Cálculo de la mediana cuando el número } \\
\text { de observaciones es impar, enunciado } \\
\text { explícito. }\end{array}$} & Logrado & $83,3^{a}$ & $80,0^{a}$ \\
\hline & No Logrado & 16,7 & 20,0 \\
\hline \multirow{2}{*}{$\begin{array}{l}\text { Cálculo promedio ponderado con enunciado } \\
\text { explícito. }\end{array}$} & Logrado & $45,2^{\mathrm{a}}$ & $46,7^{\mathrm{a}}$ \\
\hline & No Logrado & 54,8 & 53,3 \\
\hline \multirow{2}{*}{$\begin{array}{l}\text { Cálculo de promedio ponderado con enun- } \\
\text { ciado implícito. }\end{array}$} & Logrado & 7,1 & 0,0 \\
\hline & No Logrado & 92,9 & 100,0 \\
\hline
\end{tabular}

*: Letras iguales indican que no existen diferencias significativas al 5\%.

En relación con el cálculo del promedio aritmético en presencia de datos agrupados con información explicita, se observa que solamente un $45,2 \%$ de estudiantes de la Universidad 1, calculan correctamente el promedio aritmético, en cambio en la Universidad 2 el porcentaje de logro aumenta considerablemente a un 73,3\%. En este sentido los estudiantes de la Universidad 2 logran descodificar la información presente en situaciones problemáticas de lo que se infiere que estos estudiantes logran entender la estructura de resumen asociada al cálculo del promedio, presentándose diferencias estadísticamente significativas entre las instituciones formadoras ( $\mathrm{p}$-valor $<0,05$ ).

Otra habilidad que se evalúa mediante el instrumento es el cálculo de la mediana en relación al número de observaciones, que como es sabido influyen en su ubicación. En la existencia de un número impar de observaciones el 
Comprensión de las medidas de tendencia central: un estudio comparativo en estudiantes de pedagogía en matemática en dos instituciones formadoras chilenas

$83,3 \%$ de los estudiantes de la Universidad 1 realizan correctamente su cálculo, cuyo logro disminuye al $80,0 \%$ en la Universidad 2. Situación similar se da cuando el número de observaciones es par con porcentajes relativamente más descendidos del orden del 76,2\% y 73,3\% respectivamente no presentándose diferencias significativas en ambos grupos.

Como es sabido en el caso cuando las observaciones no tienen la misma importancia relativa, la media aritmética no es el mejor representante de los datos, dado que ella considera el mismo peso a cada dato. En tal sentido resulta de interés conocer el nivel de logro de los estudiantes en FID al ser sometidos a situaciones problemáticas de carácter implícito donde el índice de resumen es el promedio ponderado dado que la población se encuentra dividida en grupos o estratos no necesariamente del mismo tamaño. Se observa que solamente un $7,1 \%$ de los estudiantes de la Universidad 1 calculan el promedio ponderado, llamando la atención que en la Universidad 2 ningún estudiante de una respuesta acertada.

Cabe mencionar que una de las exigencias del sistema educacional chileno en la FID es que durante su formación los estudiantes adquieran una fuerte base teórica de los contenidos que deben ser enseñados a nivel de Educación Básica o Media. Para tal efecto en el instrumento aplicado se introdujo un reactivo utilizado originalmente por Godino (1999) en el cual se entregan las calificaciones de dos grupos denotadas con conceptos de carácter cualitativo ordinal (I=Insuficiente, A: Aprobado, $\mathrm{N}=$ Notable, $\mathrm{S}=$ Sobresaliente). Los encuestados deben responder dos interrogantes:

a) ¿Qué grupo ha obtenido mejores notas?

b) ¿Cuál sería la medida de tendencia central más apropiada para representar estos datos?

Los resultados evidencian que solamente el 46,5\% de los estudiantes dan respuesta a las preguntas. En general construyen una tabla de distribución de frecuencia y hacen un análisis comparativo del porcentaje de alumnos que obtienen cada tipo de calificación.

Además de lo anterior, los estudiantes asignan valores numéricos a cada una de las categorías enunciadas, como por ejemplo "Insuficiente" lo designan con el número uno, "Aprobado" con el número dos, "Notable" con el número tres y "Sobresaliente" con el número cuatro y a partir de dichas codificaciones dan respuesta a las interrogantes planteadas. En la siguiente tabla se muestra la distribución de los estadígrafos propuestos por los encuestados según institución. E1 84,2\% de los estudiantes de la Universidad 1 y el 71,4\% de la Universidad 
2 calculan el promedio aritmético, asumiendo un carácter cuantitativo a la variable bajo estudio.

Tabla 4 - Distribución de los porcentajes de alumnos de formación inicial docente de Pedagogía en Educación Matemática en relación a los estadígrafos propuestos, en presencia de información cualitativa ordinal.

\begin{tabular}{lcc}
\hline Estadígrafos propuestos & Universidad 1 & Universidad 2 \\
\cline { 2 - 3 } & Porcentaje & Porcentaje \\
\hline Promedio Aritmético & 84,2 & 71,4 \\
Mediana & - & 14,3 \\
Moda & - & 14,3 \\
Porcentaje & 10,6 & - \\
Promedio Ponderado & 5,3 & - \\
\hline
\end{tabular}

Otro estadígrafo propuesto para resumir la información es la mediana, donde solamente un 14,3\% de los estudiantes de la Universidad 2 explicitan que es el estadígrafo adecuado teniendo presente la naturaleza de la variable, situación similar ocurre con el valor modal. De lo anterior podemos inferir que solamente un 14,3\% de los estudiantes de la universidad 2 tienen claro la relación que existe entre los índice de resumen numérico y la naturaleza de la información de lo cual podemos inferir que en su formación no solamente se está haciendo un uso procedimental de las formulas las cuales solamente deben "alimentarlas" numéricamente, sino más bien profundizar en el contexto teórico que hay detrás de tales medidas de resumen numérico.

Otro reactivo apuntaba a que los alumnos explicaran la significación del promedio aritmético en la siguiente situación: "Según el Instituto Nacional de Estadística de Chile (INE) el número medio de hijos por familia en Chile es 1,4. Explica que significa para ti esta frase". El objetivo es que los alumnos interpreten el valor promedio a partir de sus conocimientos adquiridos en su formación estadística previa, teniendo presente que es una medida de tendencia central de carácter socialista (reparto equitativo en una distribución de datos). En general los estudiantes argumentan que el promedio se obtiene a partir de la suma de todas las observaciones divididas por el total de individuos. Los porcentajes de logro son similares en ambas universidades $40,0 \%$ y $48,0 \%$ respectivamente. De lo anterior se desprende que en general 
los estudiantes tienen arraigada una práctica discursiva orientada al cálculo algorítmico, centrando el análisis en una explicación de tipo procedimental. No obstante se esperaba que ellos fueran capaces de explicar el sentido de tal resumen estadístico en un contexto dado. Cabe destacar que los estudiantes argumentan que dicho valor al ser decimal no tiene sentido, lo cual permite inferir que los alumnos no tiene claro que el operador promedio aritmético no es una operación interna (Tabla 5).

Tabla 5 - Distribución de los porcentajes de alumnos de formación inicial docente de Pedagogía en Educación Matemática en relación a las respuestas dadas.

\begin{tabular}{lcc}
\hline \multirow{2}{*}{ Respuestas } & Universidad 1 & Universidad 2 \\
\cline { 2 - 3 } & Porcentaje & Porcentaje \\
\hline Es la suma de todas las observaciones divididas por & $40,0^{\mathrm{a}}$ & $48,0^{\mathrm{a}}$ \\
el total & & \\
No Responde & $20,0^{\mathrm{a}}$ & $22,0^{\mathrm{a}}$ \\
El promedio es 1,4 hijos & 30,0 & 20,0 \\
No tiene sentido en el contexto del enunciado & 10,0 & 10,0 \\
\hline
\end{tabular}

*: Letras iguales indican que no existen diferencias significativas al 5\%.

Ciertamente resulta paradójico constatar en este estudio que estudiantes con formación universitaria no son capaces de explicar el sentido un valor promedio en un contexto más aún si se trata de una medida de tendencia central introducida en el currículo escolar chileno desde la enseñanza básica.

Habilidades de descodificación de información numérica presente en representaciones gráficas

Los estudiantes en su Formación Inicial Docente también deben adquirir habilidades de descodificar información presente en representaciones gráfica, en particular gráficos de barras simple e histogramas para resumir la información mediante algún índice numérico. Actividades de este tipo se encuentran presente en los textos escolares entregados por el Ministerio de Educación Chileno (MINEDUC). En la siguiente tabla se presentan los porcentajes de logro de los estudiantes según institución formadora en relación a obtener estimaciones del promedio aritmético y mediana. 
Tabla 6 - Distribución de los porcentajes de logro en alumnos de formación inicial docente de Pedagogía en Educación Matemática en relación a la descodificación de información numérica en representaciones gráficas

\begin{tabular}{|c|c|c|c|}
\hline \multirow[b]{2}{*}{ Descodificación de información de un } & & Universidad 1 & Universidad 2 \\
\hline & & Porcentaje & Porcentaje \\
\hline \multirow{2}{*}{$\begin{array}{l}\text { Gráfico de barras simple, para estimar el } \\
\text { promedio aritmético }\end{array}$} & Logrado & $45,2^{a}$ & $46,7^{a}$ \\
\hline & No Logrado & 54,8 & 53,3 \\
\hline \multirow{2}{*}{$\begin{array}{l}\text { Gráfico de barras simples para estimar la } \\
\text { mediana. }\end{array}$} & Logrado & $23,8^{\mathrm{a}}$ & $13,3^{\mathrm{a}}$ \\
\hline & No Logrado & 76,2 & 86,7 \\
\hline \multirow{2}{*}{$\begin{array}{l}\text { Histograma para estimar el promedio } \\
\text { aritmético. }\end{array}$} & Logrado & $83,3^{\mathrm{a}}$ & $73,3^{\mathrm{a}}$ \\
\hline & No Logrado & 16,7 & 26,7 \\
\hline
\end{tabular}

*: Letras iguales indican que no existen diferencias significativas al 5\%.

Se puede observar que en general los alumnos de ambas universidades presentan porcentajes de logro similares en relativamente descendidos del orden del $45,2 \%$ y $46,7 \%$ respectivamente. De lo anterior se desprende que ellos tienen dificultades para asociar la altura de cada barra con las frecuencias observadas respectivas, evidenciado que podrían estar más familiarizados con cálculos algorítmicos que den cuenta del cálculo de la media aritmética.

Sin embargo cuando la información es resumida en un histograma, los resultados evidencian mayores porcentajes de logro con un 83,3\% (Universidad 1) y $73,3 \%$ (Universidad 2); situación que puede ser explicada por los aprendizajes previos vinculados a la familiarización con este tipo de representación, muy estudiada en los cursos de estadística descriptiva. Además cabe mencionar que este método gráfico se introduce tempranamente en el currículo escolar.

No obstante lo anterior los porcentajes más descendidos se presentan cuando deben realizar una estimación de la mediana, en el cual solamente un $23,8 \%$ de los alumnos de la Universidad 1 lo realizan correctamente en cambio en la Universidad 2 solamente un 13,3\%. Sin embargo las diferencias son solamente numéricas y en ningún caso estadísticas a un nivel de significación del 5\%. 
Porcentajes de logro en habilidades de simulación de distribuciones de datos bajo condiciones iniciales

Una de las habilidades a desarrollar en los estudiantes en estadística es la simulación de un conjunto de datos bajo ciertas condiciones iniciales, situación que podría permitir afianzar las propiedades de los estimadores numéricos. Así por ejemplo generar un conjunto de datos dado el promedio aritmético, como así también comparar de distribuciones de datos para develar propiedades de ellos. Este tipo de actividades permitiría introducir el concepto de variable aleatoria al término de la formación escolar. En este estudio los resultados de las habilidades de simulación según tipo de universidad se presentan en la siguiente tabla.

\section{Tabla 7 - Distribución de los porcentajes de logro en alumnos de formación inicial docente de Pedagogía en Educación Matemática en relación a la simulación y comparación de datos}

\begin{tabular}{|c|c|c|c|}
\hline \multirow[b]{2}{*}{ Habilidad } & & Universidad 1 & Universidad 2 \\
\hline & & Porcentaje & Porcentaje \\
\hline \multirow{2}{*}{$\begin{array}{l}\text { Simulación de un conjunto de datos conocido } \\
\text { el promedio ponderado. }\end{array}$} & Logrado & $45,2^{\mathrm{a}}$ & $46,7^{\mathrm{a}}$ \\
\hline & No Logrado & 54,8 & 53,3 \\
\hline \multirow{2}{*}{$\begin{array}{l}\text { Simulación una distribución de seis observacio- } \\
\text { nes (datos) a partir de un promedio dado }(4,0) \\
\text { y el mayor valor observado sea } 5,0 \text {. }\end{array}$} & Logrado & $45,2^{\mathrm{a}}$ & $40,0^{a}$ \\
\hline & No Logrado & 54,8 & 60,0 \\
\hline \multirow{2}{*}{$\begin{array}{l}\text { Comparación del logro de dos grupos de da- } \\
\text { tos aplicando medidas de tendencia central y } \\
\text { variabilidad }\end{array}$} & Logrado & $59,5^{\mathrm{a}}$ & $73,3^{b}$ \\
\hline & No Logrado & 40,5 & 26,7 \\
\hline \multirow{2}{*}{$\begin{array}{l}\text { Simulación de un conjunto de datos conocido el } \\
\text { promedio en un situación problemática }\end{array}$} & Logrado & $65,0^{a}$ & $67,0^{\mathrm{a}}$ \\
\hline & No Logrado & 35,0 & 33,0 \\
\hline \multirow{2}{*}{$\begin{array}{l}\text { Identificación de la medida de tendencia central } \\
\text { más adecuada de un grupo con información } \\
\text { explicita }\end{array}$} & Logrado & $24,0^{a}$ & $7,0^{\mathrm{b}}$ \\
\hline & No Logrado & 76,0 & 93,0 \\
\hline
\end{tabular}

*: Letras iguales indican que no existen diferencias significativas al 5\%.

Se observa que el 45,2\% de la Universidad 1 y el 46,7\% de la Universidad 2 logran simular un conjunto de datos conocido el promedio ponderado, no presentándose diferencias significativas entre las instituciones formadoras. Por otra parte cuando se solicita generar un conjunto de datos a partir de un prome- 
dio dado y con la condición que el mayor valor observado sea 5 , solamente un $45,6 \%$ de los estudiantes de la Universidad 1 lo realizan correctamente, siendo más descendido en la Universidad 2 con un porcentaje de un 40,0\%.

Sin embargo cuando se solicita realizar una simulación bajo la condiciones de un promedio conocido, los resultados arrojan mayores porcentajes de logro del orden del $65,0 \%$ en la Universidad 1 y $67,0 \%$ en la Universidad 2. Una explicación plausible es el tipo de situación enunciada que al considerar un promedio aritmético simple permite generar asociaciones con actividades habituales del sistema escolar.

Finalmente la evaluación consideró un ítem sobre la comparación de dos distribuciones de datos de manera gráfica solicitando decidir cuál de ellas presentaba un mejor rendimiento. El 73,3\% de los estudiantes de la Universidad 2 explicitan que para dar respuesta se debe considerar la variabilidad de las distribuciones de datos y no solamente el promedio aritmético. Argumentación realizan el 59,5\% de los estudiantes de la Universidad 1. En este caso se presentan diferencias estadísticamente significativas al 5\% entre los alumnos de la Universidad 1 y Universidad 2 así como también en la habilidad de identificar la medida de tendencia central más adecuada de un grupo con información explicita.

\section{Discusión de los datos y conclusiones}

En nuestro país, las medidas de resumen numérico (promedio aritmético, mediana y moda) son introducidas a temprana edad en el currículo de Educación Básica y Media y por lo mismo, se espera que los docentes desarrollen habilidades estadísticas en sus alumnos desde el inicio de su escolarización (MINEDUC, 2011, 2012a, 2012b). Por tanto se supone que los docentes de Matemática poseen habilidades para descodificar información, representar y simular distribuciones de datos entre otras, pues sabido es que no se puede enseñar lo que no se domina (HERNÁNDEZ; SANCHO, 1996).

En ese contexto, el problema indagado en el presente estudio fue conocer cuán desarrolladas tienen las habilidades de descodificación de información presentada en enunciados problemáticos, los estudiantes que se encuentran en etapa de FID, para aplicar procedimientos de cálculo y simulación estadística. Los principales resultados y conclusiones del estudio se describen a continuación:

- En general, los estudiantes presentan resultados descendidos en la habilidad para descodificar información presente en representaciones 
Comprensión de las medidas de tendencia central: un estudio comparativo en estudiantes de pedagogía en matemática en dos instituciones formadoras chilenas

gráficas y al mismo tiempo son capaces de realizar principalmente cálculos numéricos sin profundizar en la teoría que subyace en las propiedades de los estadígrafos de tendencia central presentes en el estudio. Lo anterior daría cuenta del trabajo algorítmico que han adquirido los estudiantes de FID en su ciclo de escolarización, sin mayor reflexión sobre los significados de los estadígrafos en contextos.

Cabe señalar que al resumir información mediante medidas de tendencia central, se debe tener presente que dichos estadígrafos están relacionados con la naturaleza de la variable como así también en la distribución de los datos (forma), habilidades insuficientemente desarrolladas según los resultados presentados en el estudio.

- En este sentido los datos evidencian que los estudiantes, en general, obtienen porcentajes de logro menos descendidos cuando calculan el promedio aritmético en presencia de información explicita con datos agrupados (tablas de distribución de frecuencia), sin embargo cuando los datos no tienen la misma importancia relativa el promedio aritmético no es el índice numérico más adecuado y en tal caso se debe hacer uso del promedio aritmético ponderado, en tal sentido cuando se explicita el cálculo de dicho índice en general el porcentaje de logro de ambas universidades es del 46,0\% aproximadamente.

No obstante cuando los alumnos deben calcular el promedio ponderado con enunciado explicito solamente el 7,1\% de la Universidad 1 logra realizar la tarea correctamente, de lo que se puede inferir que los estudiantes en general presentan habilidades procedimentales siempre y cuando se solicite de manera explícita el cálculo de algún índice de resumen.

- El escaso desarrollo de la habilidad de descodificación se evidencia mayoritariamente cuándo se trata de información resumida mediante representaciones gráficas. En tal sentido, los resultados del presente estudio, muestran que los estudiantes en FID pueden descodificar información de un histograma para el cálculo del promedio y no cuando la información es presentada en un gráfico de barras simples. Ello se puede deber a que los profesores del sistema escolar tienden a restarle importancia a las representaciones asociadas a datos cualitativos y centran su atención en las representaciones gráficas asociadas a información cuantitativa como son los histogramas. 
- La diferencia entre algunas habilidades menos desarrolladas entre los estudiantes de las careras bajo estudio se podrían explicar por factores de carácter contextual inherentes al aprendizaje de la estadística, tales como las concepciones previas, metodologías de enseñanza aprendizaje, las tareas de evaluación formativa y las características de la formulación de reactivos en el proceso de ejercitación. Estos asuntos no han sido considerados en el presente estudio dada la naturaleza de sus objetivos, sin embargo cabe señalar que es de interés ahondar en ellos en investigaciones futuras.

- Coherentemente con otros estudios (BATANERO, 2000), el desarrollo de habilidades algorítmicas en el sistema escolar conlleva a que los estudiantes codifiquen información no numérica como si tuviesen propiedades numéricas de facto. Así pues, en el presente estudio, los encuestados codifican de manera numérica cuando se entrega información de naturaleza cualitativa ordinal a fin de resumir mediante el promedio aritmético.

En síntesis los estudiantes en FID tienden a descodificar información entregada en enunciados o representaciones gráficas, ya sea de naturaleza cualitativa o cuantitativa, como expresiones numéricas para realizar actividades tendientes a potenciar su cálculo mediante procedimientos algorítmicos. En consecuencia es posible plantear lo siguiente:

- Los estudiantes en Formación Inicial Docente de Pedagogía en Matemática tienen anclado cognitivamente los procesos de cálculo algorítmico que les impide ir más allá del número o resumir la información en contextos, para descodificar la naturaleza de lo representado estadísticamente.

- Según el presente estudio los futuros docentes tendrían un dominio parcial de las habilidades a enseñar para realizar razonamiento estadístico y para descodificar la naturaleza de la información representada. Esto traería como consecuencia que se seguiría reproduciendo una cultura escolar de la enseñanza de la estadística basada en el cálculo algorítmico.

- Por lo mismo, pareciera, que lo razonable es que en la Formación Inicial Docente se rompa este hábito de cálculo algorítmico de los estudiantes y se tienda a desarrollar habilidades de razonamiento que les permita 
descodificar la información en su contexto y, por ende, interferir la naturaleza de los datos-variables para el correcto cálculo o representación. Lo anterior, supondría que un aspecto a explorar conjuntamente es la formación didáctica de los estudiantes en FID, a fin de responder las siguientes interrogantes: ¿qué estrategia didáctica facilita el razonamiento estadístico? y ¿qué currículo, estructura o enfoque curricular para la FID facilitará la adquisición de dichas habilidades?

\section{Referencias}

ALVEAL, Francisco Rodríguez; RUBILAR, Pedro Rodrigo Sandoval. Habilidades de codificación y descodificación de tablas y gráficos estadísticos: un estudio comparativo en profesores y alumnos de pedagogía en enseñanza básica. Avaliação, Campinas; Sorocaba, v. 17, n. 1, p. 207-235, mar. 2012.

BAKKER, A.; GRAVEMEIJER, K. P. E. Learning to reason about distribution. En: GARFIELD, J.; BEN ZVI, D. (Eds.). The challenge of developing statistical literacy, reasoning and thinking. Dordrecht, The Netherlands: Kluwer, 2004. p. 147-168

BATANERO, C. Significado y comprensión de las medidas de posición central. Uno. Revista de Didáctica de las Matemáticas, Barcelona, n. 25, p. 41-58, 2000.

BEHAR, R; OJEDA, M. El proceso de aprendizaje de la estadística: ¿Qué puede estar fallando? Heurística, Colombia, v. 10, p. 26-43, 2000.

\section{CAMPBELL, S. K. Flaws and fallacies in statistical thinking. New} Jersey: Prentice-Hall. 1974.

COBO, Belén. Significados de las medidas de posición central para los estudiantes de secundaria. 2003. $301 \mathrm{f}$. Tese (Doutorado) - Curso de Curso de Didáctica de La Matemática, Universidad de Granada Departamento de Didáctica de La Matemática, Granada, Granada, 2003.

COBO, B; BATANERO, C. Significados de la media en los libros de texto de secundaria. Enseñanza de las Ciencias, Barcelona, v. 22, n. 1, p. 5-18, 2004. 
GAL, I. Adult's statistical literacy. Meanings, components, responsibilities. International Statistical Review, Netherlands, v. 70, n. 1, p. 1-25. 2002.

GALICIA, Silvia Azucena Mayen. Comprensión de las medidas de tendencia central en estudiantes mexicanos de educación secundaria y bachillerato. 2009. 376 f. Tese (Doutorado) - Curso de Didáctica de La Matemática, Universidad de Granada Departamento de Didáctica de La Matemática, Granada, 2009.

GARCÍA Cruz, J. A.; GARRETT, A. J. Understanding the Arithmetic Mean: a study with secondary and university students. Journal of the Corea Society of Mathematical Education, Series D: Research in Mathematical Education, Corea, v. 12, n. 1, p. 49-66, 2008.

GARCÍA, I.; GARCÍA CRUZ, J. A. La media aritmética. Form. Profesorado Investig. Educ. Mat., España, v. 6, p. 197-217. 2004.

GATTUSO, L; MARI, C. Development of concepts of the arithmetic average from high school to university. En: PUIG, L.; GUTIÉRREZ, A. (Eds.). Proceedings of the XX Conference on the Psychology of Mathematics Education. Universidad de Valencia, v. 2, p. 410-408, 1996.

GODINO, J. D. Análisis epistémico, semiótico y didáctico de procesos de instrucción matemática. Trabajo presentado en el grupo de trabajo "Didáctica de la matemática como disciplina científica". En: SIMPOSIO DE LA SOCIEDAD ESPAÑOLA DE INVESTIGACIÓN EN EDUCACIÓN MATEMÁTICA (SEIEM), 3., 1999, Valladolid, España. [Anais...]. Valladolid, España, 1999.

HERNANDEZ, F.; SANCHO, J. Para enseñar no basta con saber la asignatura. Barcelona: Paidós, 1996.

HOLMES, P. Teaching Statistic. Sloug: Foulsham Educational, UK, p.1116,1980 .

MCMILLAN, J. H; SCHUMACHER, S. Investigación educativa. Madrid: Pearson, 2007.

MEVARECH, Z. R. A deep structure model of students' statistical misconceptions. Educational Studies in mathematic, Springer Netherlands, v. 14, p. 415-429, 1983. 
Comprensión de las medidas de tendencia central: un estudio comparativo en estudiantes de pedagogía en matemática en dos instituciones formadoras chilenas

MINISTERIO DE EDUCACIÓN DE CHILE - MINEDUC. Objetivos fundamentales y contenidos mínimos obligatorios enseñanza media. 2009

MINISTERIO DE EDUCACIÓN DE CHILE - MINEDUC. Estándares Orientadores para egresados de carreras de Pedagogía en Educación Básica. 2011.

MINISTERIO DE EDUCACIÓN DE CHILE - MINEDUC. Gobierno de Chile. Decreto N. 439 - Bases Curriculares Educación Básica. 2012a. MINISTERIO DE EDUCACIÓN DE CHILE - MINEDUC. Estándares Orientadores para carreras de Pedagogía en Educación Media. 2012b.

MOCHÓN, Simón; TLACHY, Anell, María Margarita. Un estudio sobre el promedio: concepciones y dificultades en dos niveles educativos. Educación Matemática, México, p. 5-28, dic. 2003.

MOORE, D. S. Teaching statistics as a respectable subject. En: GORDON, F.; GORDON, S. (Eds.). Statistics for the twenty-first century. Washington, Mathematical Association of America, p. 14-25, 1991.

PLACKETT, R. L. The Principle of the Arithmetic Mean. In: PEARSON, E.; KENDALL, M. G. Studies in the History of Statistics and Probability. London: Griffin. 1970. v. 1.

REICH, Ricardo. Convenios de Desempeño 2011-2014. In: SEMINARIO SCT-CHILE INNOVACIÓN Y ARMONIZACIÓN, 1., 2011, Pucon. Presentación. Pucon: Gobierno de Chile, 2011. p. 1 - 41.

RODRIGUEZ, E. Tendencias en las políticas educativas latinoamericanas. Pensamiento educativo, Santiago, v. 17, n. 2, p. 133- 152, 1995.

RUSSELL, S. J.; MOKROS, J. R. What's typical?: Children's ideas about average. In: D. VERE-JONES, D. (Ed.) Proceedíngs of the Third International Conference on Teaching Statistics. Voorburg, The Netherlands: International Statistical Institute, 1991. p. 307-313.

SHAUGHNESSY, J. M. Research on statistics learning and reasoning. En: LESTER, F. (Ed.). Second handbook of research on mathematics teaching and learning. Greenwich, CT: Information Age y NCTM, 2007. p. 957-1010. 
STRAUSS, S.; BICHLER, E. The development of children's concepts of the arithmetic average. Journal for Research in Mathematics Education, Virgina, USA, v. 19, n.1, p. 64-80. 1988.

UNESCO. 50 Years for Education [en línea]. 1997.

Agradecimientos: Los autores agradecen a los evaluadores del artículo, por sus valiosos comentarios y sugerencias que han permitido mejorar la comunicación de los resultados del estudio.

Francisco Enrique Rodriguez Alveal - Universidad del Bio-Bio Chile Chillan | Chile. Contacto: frodriguez@ubiobio.cl

Pedro Rodrigo Sandoval Rubilar - Universidad del Bio-Bio Chile Chillan | Chile. Contacto: psandoval@ubiobio.cl

Ana Carolina Maldonado Fuentes - Universidad del Bio-Bio Chile Chillan | Chile. Contacto: amaldonado@ubiobio.cl

Artigo recebido em 17 de abril de 2015 e aprovado em 7 de março de 2016. 